\title{
Embedded Based Smart, Secured and Safe Vehicle System
}

\author{
Prof. R.K. Moje ${ }^{1}$, Mrugesh Bannatwale ${ }^{2}$, Sagar Jamdade ${ }^{3}$, Preeti Halloli ${ }^{4}$ \\ Faculty, Department of Electronics, PDEA's College of Engineering, Manjri (Bk), Pune, India ${ }^{1}$ \\ Student, Department of Electronics, PDEA’s College of Engineering, Manjri (Bk), Pune, India 2, 3,4
}

\begin{abstract}
In today's fast and crowded world, road accident is a major concern. The highest fatal traffic accidents occur on the curved roads at night time. Also the glare problem due to focus of headlight is dangerous. Even the improper indications given to the nearby vehicle increases chances of fatal accidents. These facts indicate the importance of advancement in conventional vehicle systems. This paper explains the prototype model of vehicle system consisting of intelligent headlight intensity control, adaptive headlights, auto indicator-off system, anti-collision system and vehicle to vehicle communication. Proposed system is very cost efficient and reliable and can be mounted on any vehicle, it is specially focused to increase the safety and security of low-end vehicles.
\end{abstract}

Keywords: Adaptive Headlight, Headlight intensity, Anti-collision, Indicator, Curved Road, Servo motor.

\section{INTRODUCTION}

Our proposed system is based on producing safe and secured vehicle system. Nowadays we hear almost every day about fatal road accidents. Even our Prime Minister Narendra Modi appealed people to come forward to prevent fatal road accidents in his famous radio show "maan ki baat". This indicates the severity of issue. Survey shows that there is one death after every four minutes due to road accidents in India [9].

Major causes for road accidents includes decreased visibility of driver on curved roads during night, glare of headlight, incorrect indication to the nearby vehicles, etc. During night time, on curved roads when driver takes turn the headlight focus cannot trace the curved angle of roads hence, driver can't see the other side of road. On other side of turn there may present other vehicle or pedestrian, so there is high chance of accident. Again the glare problem due to high intensity of headlight makes it impossible for driver to see anything in front of him, this is very dangerous situation. Also sometime driver may forget to turn off the indicator this may give wrong indication to the vehicle preceding or ahead of that vehicle. Now-a-days private taxis are in boom and it has raised the question of security of passenger, especially for women, for instance recently occurred Uber taxi cases in New Delhi.

In order to deal with all the problems explained above we have proposed a smart vehicle system solution. There are many scenarios we have considered for our application as follows,

- Adaptive steerable headlight system.

- Headlight intensity control.

- Automatic indicator off system.

- Anti-collision mechanism.

- Vehicle to Vehicle communication.

In adaptive headlight system the headlight focus will trace the curve roads which will make the other side of curve road visible to the driver. Headlights will be connected to the servo motor which will be controlled via microcontroller output. Microcontroller output will be dependent on input from pot which is connected to the steering wheel. Thus during steep turn as driver will rotate its steering wheel headlights will also move accordingly and this will illuminate other side of the turn. Again these smart headlights will turn on automatically by sensing the surrounding luminosity to improve visibility of driver in conditions like; night time driving, twilight, rain, tunnels and other dark places. This will also save driver's effort to switch headlights manually, which he may forget sometime. LDR (light dependent resistor) is use for this application. Next to deal with the glare problem intensity of headlight can be controlled automatically using LDR. Automatic dimmer and upper mode will be adjusted when any vehicle passes from front side so that headlight focus will not cause glare problem for driver of front vehicle. To avoid false indication we are introducing auto indicator off system. We are also including anti-collision system with the help ultrasonic sensor which will continuously sense the distance using Doppler Effect as well as headlight intensity of front vehicle, and when the distance between two vehicles decreases below specific level and intensity increases above specific level the vehicle will first give indication and later automatically apply brakes for accident avoidance. And finally we have introduced the vehicle to vehicle communication to communicate using RF (radio frequency).By using radio frequency one can send massage/help massage/or simply SOS massage to nearby vehicle. The other vehicle will receive the massage by RF receiver.

\section{LITERATURE SURVEY}

Road accident is a major problem our nation is facing today. Survey reports by National Crime Records Bureau, Ministry of Road Transport \& Highway, and Law commission of India, Global status report on road safety 2014 shows following statistics-

- 16 children die on Indian road daily

- One death every four minutes due to road accident 
- One serious accident per minute and 16 die every hour

- 1214 crashes everyday \& 377 die every day [9].

Even if headlights are supposed to help the driver in driving, it may cause glaring problem. According to the SAE (Society of Automotive Engineers) rule of automotive illumination, in dim mode it should be 700 lumens and in bright mode it should be 1200 lumens, when tested away from 50 feet from vehicle [1].Study shows that if our eyes are exposed to bright light source more than 1000 lumens even from 20 feet, we experience a glare, due to over exposure of rods and cones inside our eye. Even after source of glare is removed, after imaging remains in our eye that creates a blind spot. This is called as Troxler effect, causing temporary blindness for driver and this increases driver's reaction time by 1.4 seconds, which is enough to cause a fatal accident. Any one exposed to sudden bright light experiences this Troxler effect [3].

Also many accidents occurs on roads having steep turns and curved roads in hilly areas, due to presence of blind spots, These areas cannot be seen directly by looking forward or by looking through either of side mirrors. Many reasons causes these blind spots like steep curves in roads, weather conditions, poor infrastructure, improper street lights etc. which creates problems for driver. A vehicle with normal headlights sends the light rays tangential to curves; this reduces the ability of driver to see the blind spots. These blind spots can be eliminated for safe driving using adaptive headlight system [2].

Many people lost their lives every year in vehicle collision majorly due to driver's inability to observe keenly in vehicle's vicinity while driving and in traffic condition, while overtaking, sudden braking on turns, loss of control etc. There are certain research works carried out on anticollision devices using ad hoc wireless network, V2V communication, GPS and radar implementation but all focuses on communicating the driver and later he will take action manually which includes chances of collisions[4]. But we have developed a system which will not only provide driver a safe warning but also automatically apply braking system after specific time if any sudden action not taken by driver.

Now-a-days private taxi services have been increased to huge number so it has raised the question of security especially for women passenger. Recently we have heard lot of news about such cases specially that happen with Uber taxi few months ago. So for security purpose of passenger we have devised a system to communicate with nearby vehicles and sending message for help.

The concept proposed by us is not new in high end car like Volvo, BMW, Audi etc. where in these mechanisms are already employed but they have followed different approach in doing so. These vehicles use expensive sensors to measure speed, steering angle and yaw. Due to use of such sophisticated devices in these high end cars the cost of this simple module is as high as $\$ 1000$. For instance following table shows cost of Adaptive headlight system in various cars [2].
Table1. Cost estimate of AHS in high-end cars

\begin{tabular}{|c|c|c|}
\hline Serial no. & Car model & Cost estimate \\
\hline 1. & $\begin{array}{c}\text { Mercedes Benz } \\
\text { S520 }\end{array}$ & $\$ 700$ \\
\hline 2. & $\begin{array}{c}\text { Mercedes Benz } \\
\text { Premiun 3 engine }\end{array}$ & $\$ 6550$ \\
\hline 3. & Volvo V60 & $\$ 850$ \\
\hline 4. & Lexus RX350 & $\$ 515$ \\
\hline
\end{tabular}

From above table it is clear that cost of this module is very expensive in high-end vehicles due to use of sophisticated devices, whereas according to our proposed system it will cost much less. We have targeted low-budget cars like Tata Indica, Maruti Swift etc. which are commonly found on Indian roads.

\section{DESCRIPTION}

\section{BLOCK DIAGRAM DISCRIPTION}

The system can be represented using block diagram as shown below.

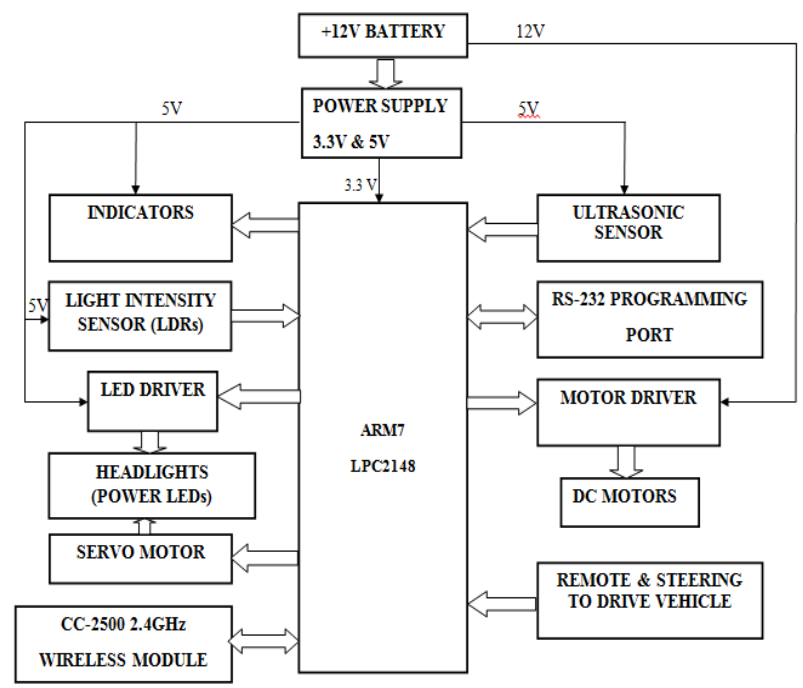

Fig1. Block diagram of smart vehicle system

This work is aimed at producing a cost effective smart, secured and safe vehicle system. We have built a prototype model which as shown in above block diagram. Following is the hardware description.

1. ARM-7 module - In our system we are using LPC 2148. It acts as the major controller unit of the system. Input from various sensors like ultrasonic, LDR, RF module, steering pot/potentiometer is given to this unit on which it process according to the programming and gives output to the driver circuits of indicator, vehicle brake, servo motor etc. It needs $3.3 \mathrm{~V}$ to drive the ARM7 module.

2. Power supply unit- On-board $12 \mathrm{~V}$ rechargeable battery is use to drive dc and servo motors. To drive ARM7 we require $3.3 \mathrm{~V}$ and for various sensors and we require $5 \mathrm{~V}$ to drive RF module, LDR etc. So to do this we will have to build variable power supply unit using voltage divider circuit and regulator ICs like LM7805 and LM1117.

3. Steering system - It will include a steering wheel attached to potentiometer. As steering angle changes 
potentiometer resistance vary which is given as ADC input 2. WORKING

to the microcontroller, which further drives the servo Module-wise working is explained below;

motor attached to the axel using PWM.

4. Indicators - There will be two indicators. They are driven by $5 \mathrm{~V}$ supply connected via resistor to limit the current. They are also connected to the ARM module so as to switch off it automatically after taking turn, after specific time.

5. LED drivers- Required for interfacing low-level logic circuitry and Power LEDs. ULN 2803 high voltage, high current Darlington arrays are used.

6. Light intensity sensor- We are using LDRs here to sense the ambient light intensity as well as intensity of light coming from headlight of other vehicles. The output of LDR is given to microcontroller and indirectly the head light intensity is control by LDR. One LDR will be mounted on windshield to measure surrounding ambient luminosity.

7. Headlight- For headlights we are using power LEDs. There are four power LEDs used. Two are used for high beam of light having high luminosity and two are for dip beam having low luminosity.

8. Ultrasonic sensor- Ultrasonic sensor is used for anticollision system. It will sense the distance of nearby vehicle and other objects, if this distance decreases beyond specific limit it, will inform the controller and controller will take quick action and will warn by ringing buzzer and will apply brakes automatically if no manual action is taken within specific time.

9. Servo motor- To drive and control the adaptive motion of headlights attached to axel connecting front wheels. Servo Motor gives proper angle-wise motion. Servo motor is having self-feedback circuit which helps for accurate movement.

10. DC motor- Simple dc motors are being used as wheels of the prototype model. They are driven by $12 \mathrm{~V}$ on board battery supply.

11. Motor Driver- It is required to drive the motor properly and it provides the required supply voltage and current to motors to drive it in either direction. L293d is used for this purpose; it works on H-bridge principle.

12. RF Module- In our system we are using CC2500 RF module. The RF module is use to communicate with nearby vehicles. In our system we are using $2.4 \mathrm{GHz}$ radio frequency range.

Components List

- LPC2148 controller

- Ultrasonic sensor HC-SR04

- Power LEDS

- Servo motor

- Motor driver IC-L293D

- LDR

- CC2500 RF Module

- Potentiometer

- LCD

- LM324 amplifier IC

Software's Used

- Proteus 8

- Keil Micro vision 4
1. Adaptive headlight- In current vehicle system whenever a vehicle takes steep turn over curved road in night mostly suffers low visibility problem. But in our system we have overcome it by making head lights to move in accordance to steering. The steering is connected to potentiometer as steering moves potentiometer changes its resistance value. Thus output voltage obtained also varies. This value is first converted into digital form with the help of ADC and given to microcontroller. Head light is connected to servo motor which is connected to microcontroller. As steering moves, potentiometer value gets changed which changes servo motor angle by some value hence headlights will also moves specific angle. And they will trace the curved path and driver can see even the blind spots successfully.

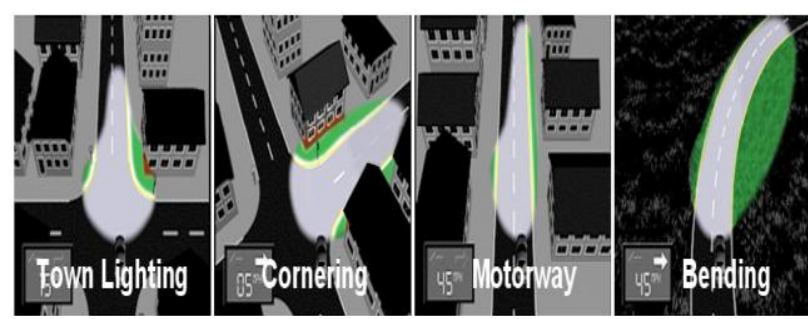

Fig2. Proposed Adaptive Headlight System

2. Headlight intensity control- Firstly LDR on the windshield will sense the luminosity of surrounding. If it is less than specific value the headlight will automatically will turn on. Luminosity decreases during night time, inside tunnel, subways etc. So driver will not have to worry about turning on and off of headlight manually. Again to deal with glare problem we have two LDRs near each headlight in order to detect light intensity coming from headlights of vehicles coming from front side. If this intensity is more than specific value and distance measured by ultrasonic sensor is less than threshold value then automatic dim beam will glow and high beam will remain off. Once the vehicle passes by again high beam will glow, this again saving driver's effort to manually switch upper/dipper headlight.

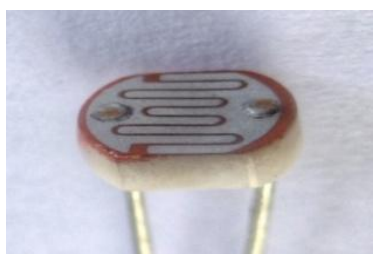

Fig3. LDR

2. Auto indicator off mechanism- Many times we turn ON the vehicle indicator and forgot to turn it OFF, this gives false indication to other vehicle. To avoid this false indication we are introducing auto indicator off system. After taking turn successfully, the steering angle will be monitored continuously. If it is found near around zero constantly for specific time indicators will turn off automatically. This will save driver's effort to turn it off manually which is not done each time manually by driver. Obviously there will be manual option also available to turn indicators ON/OFF also. 
3. Anti-Collision Mechanism- A microcontroller receives echo signals from ultrasonic range finding sensor. This information is used to excite vehicle's breaking system. Ultrasonic sensors continuously read distance between front object and the vehicle. If the distance reduces to certain level, controller will take quick action and will warn by ringing buzzer and will apply brakes automatically if no manual action is taken within specific time. For our prototype we are using HC - SR04 ultrasonic ranging module, the basic principle of work:

- Using IO trigger for at least 10us high level signal,

- The Module automatically sends eight $40 \mathrm{kHz}$ and detect whether there is any pulse signal back.

- If the signal is back through high level, then the time of high output IO duration is the time from sending ultrasonic signal to its returning.

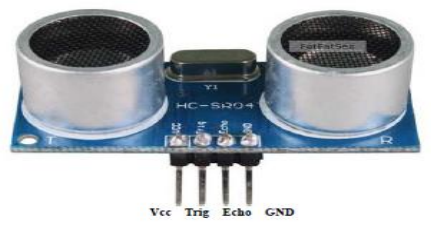

Fig4. Ultrasonic Sensor

4. Vehicle to Vehicle communication- We have achieved this using CC2500 RF module. Vehicles current status will be continuously notified to nearby vehicles within specific range about direction of moving, taking turns, speed, sudden applied brakes etc. Along with this we have introduce one security option for passenger, if he/she feels any problem, insecurity he/she can suddenly press the dedicated switch and all nearby vehicles will be informed suddenly along with SOS help signal and alarm system, so nearby passing vehicles can provide immediate help. We are designing a vehicle to vehicle communication for security application using RF communication module. We are using CC2500 $2.4 \mathrm{GHz}$ RF Transceiver It is an FSK Transceiver module, which is designed using Chipcon IC (CC2500). It is a true single chip transceiver; it is based on 3 wire digital serial interface and an entire Phase Locked Loop for precise local oscillator generation so the frequency could be setting. It can be used in UART/NRZ/Manchester.

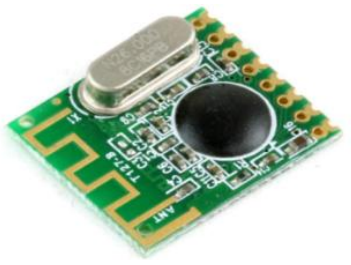

Fig5. CC2500 2.4 GHz RF Transceiver

\section{ADVANTAGES}

- Automatic controlling of headlights and indicators.

- It can be easily mounted on the any cars and sport bikes as a simple device.

- Increases visibility of the driver on curved roads and intersections.

- Response time of driver becomes good.
- It is a cost effective solution for dealing with night time accidents occurring on the curved roads and intersections.

- Increased safety for drivers, pedestrians and passenger.

- Fully automotive system thus reduces human efforts and is a robust system.

\section{APPLICATIONS}

- In Automobiles Four wheeler

- In two wheeler like sports bikes etc.

- Vehicles required for smart city.

\section{CONCLUSION}

Embedded based smart, secured and safe vehicle system serves as a reliable and efficient system for driving at sharp turns during night. The system is inexpensive, simple and dependable assembly. This system provides the ability to illuminate the road at sharp turns or corners continuously corresponding to angular rotation of sensor which is attached to the steering. Auto indication off system helps in false indication. Due to increasing traffic, anti-collision system taking major role to avoid accidents. Also intelligent vehicle to vehicle communication using cc2550 is to be implemented. Every measure to avoid accidents is taken in our system. And most importantly whole work is focused towards low-end vehicles hence a low budget and reliable system. Thus implementation of this device in all the vehicles in future will not only avoid accidents but also provide safe and comfortable driving. Hope so all of us will travel through the vehicle equipped with our proposed model.

\section{ACKNOWLEDGEMENT}

We acknowledge the efforts by the experts who have contribution towards the development of the different automation systems. We acknowledge the help and suggestions by our teachers and guide. We also acknowledge the support given by the reviewers of the journal for modifications and suggestions to improve the quality of paper.

\section{REFERENCES}

[1] Mr. K. kaliyrasu Dr. C. Karthikeyan: "Design of an Automotive Safety System using Controller Area Network" IEEE International Conference on Robotics, Automation, Control and Embedded Systems, 18-20 February 2015 [4432-4436].

[2] Shreyas S, Kirthanaa Raghuraman, Padmavathy AP, S Arun Prasad, G. Devaradjane: "Adaptive Headlight System for Accident Prevention" IEEE International Conference on Recent Trends in Information Technology -2014, [111-117].

[3] Muralikrishnan. R: "Automatic Headlight Dimmer a Prototype for Vehicles", IJRET-Volume 3, Feb-2014[85-90].

[4] Jyotiraman De: "Universal Adaptive Headlight System" IEEE International Conference on Vehicular Electronics and Safety, December 16-17, 2014, [7-10].

[5] Shival Dubey and Abdul Wahid Ansari: "Design and Development of vehicle anti-collision system using Electromagnetic and Ultra sonic Sensors" ISSN: 2319-3182, Volume-2, Issue-1, 2013 [82-83].

[6] Dhruv Gupta, Gunvantsinh Gohil, Mehul S Raval:" Driver Friendly Headlight Controller for Driving in Developing Countries", ISSN 2231-5381 vol 8 issue 10-dec 2012, [568-573].

[7] Victor Nutt, Shubhalaxmi Kher, Mehul Raval: "Fuzzy Headlight Intensity Controller using Wireless Sensor Network", ICNICT, vol. 2, November 2009.[17-20]. 